\title{
The design of the vehicle location system based on wireless sensor network
}

\author{
FANHENG \\ JILIN TEACHER'S INSTITUTE OF ENGINEERING \&TECHNOLOGY
}

Keywords: Wireless, Vehicle ,Node.

\begin{abstract}
Vehicle tracking based on wireless sensor is proposed in this paper the design of the positioning system, using wireless sensor network with low cost, low power consumption, multi-function, the characteristics of the sensor nodes deployed in monitoring area, through the vehicle's sound, vibration, infrared, magnetic field, such as signal analysis, to identify location and tracking, and in appropriate time to continue to track vehicles.
\end{abstract}

\section{Introduction}

As wireless communications, integrated circuits, sensors and mems technology rapid development and increasingly mature, low cost, low power consumption, multi-function of the mass production of micro sensor possible. These sensors are usually in small volume integrates information collection, data processing and wireless communication and other functions. Wireless sensor network (WSN) is deployed in monitoring area of a large number of micro sensor nodes through wireless communication form a multiple hops self-organizing network, its purpose is collaboration awareness, collection and processing network coverage area by monitoring object information, and sent to the observer. The network is a kind of no nodes at the centre of the distribution system, through the way of random delivery, a number of sensor nodes are densely deployed in monitoring area, these sensor nodes integrated with sensors, data processing unit and communication module, through the wireless channel is linked together, self-organizing network system. Is proposed in this paper based on wireless sensor network technology design of track vehicle positioning system, the system design is low cost, the advantages of high positioning precision and accuracy, and is suitable for a wide range of applications in traffic monitoring system.

\section{System architecture}

Vehicle tracking system based on wireless sensor network is composed of four layers structure: wireless sensor network (WSN), base station, the server and client. By an on-board wireless sensor nodes and testing nodes. Vehicular node is installed on the vehicle active communication device, periodically sends its own information, for detecting node detection. Detection node arrangement of distributed on both sides of highway network, detection of vehicle information, after dealing with the data through multiple hops forward testing information will be sent to the base station, a base station receive vehicle information from the sensor network, data fusion, send the result to the Internet. The server from the Internet to get the data, the database record and query and provide services. The client via cable or wireless network server, query the vehicle information, vehicle trajectory. Overall system structure as shown 


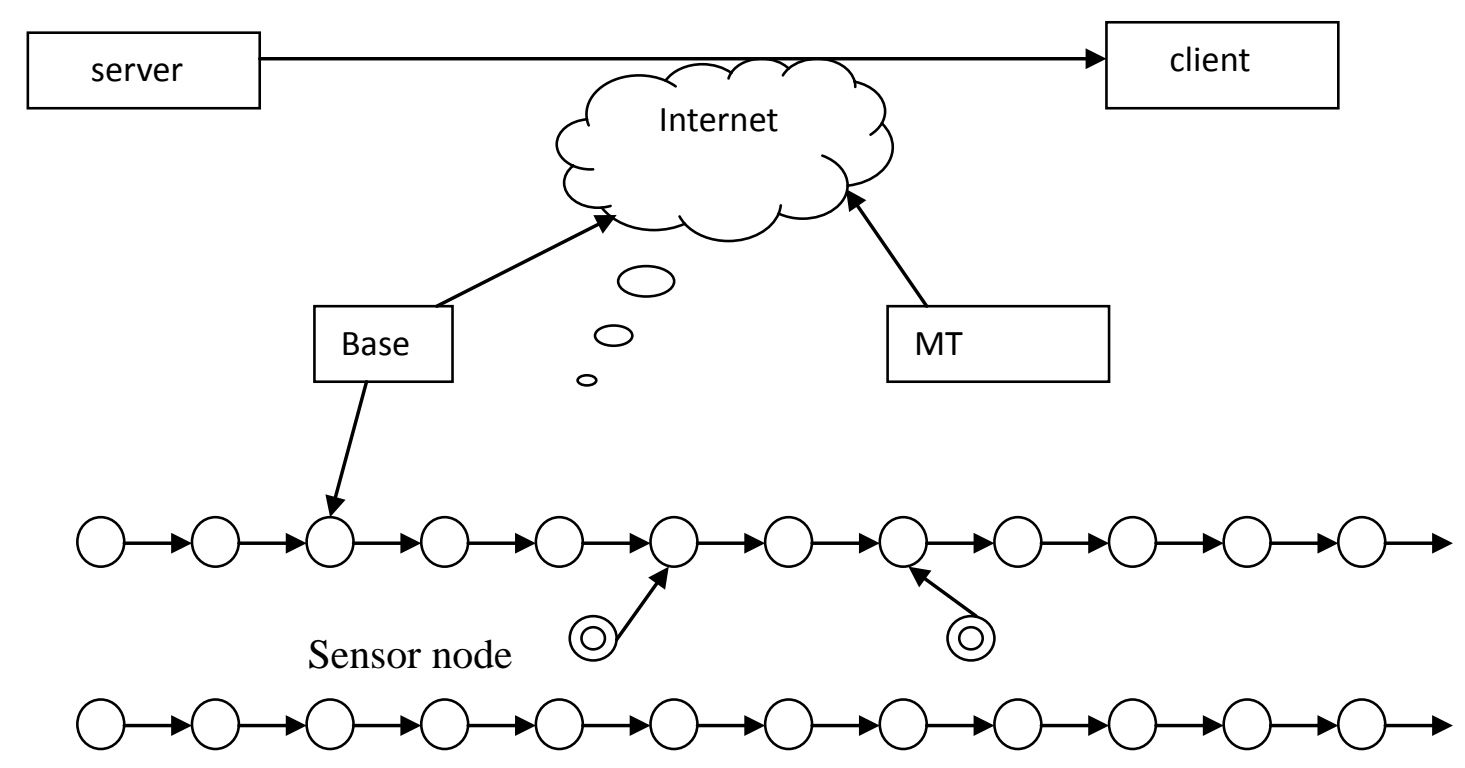

Figure 1

\section{The design of the vehicle tracking system}

System USES the IEEE802.15.4 / ZigBee network, to a large number of sensor nodes deployed in monitoring area of no center network, realize the real-time monitoring of vehicles, by the vehicle's sound, vibration, infrared, magnetic field, such as signal analysis, to identify location and tracking, and in appropriate time to continue to track vehicles.

When the vehicle is not in the monitoring area by some nodes in wireless sensor network (WSN) take turns to work, for periodic monitoring of relevant data. At this time, the wireless sensor network (WSN) is equal to the application in the environmental condition monitoring; When detect the vehicle be or get out of danger, immediately aroused around the node, to form a temporary clusters, and the cluster head according to the distribution of the follow-up of the tasks to some nodes according to the circumstance and awaken these nodes into target tracking state; Cluster head is responsible for collecting data, the information fusion, maintenance to participate in the activities of the trace nodes and nodes of a list, and will get through the network transmission of information to the management center, when the vehicle movement to the outside of the initial cluster boundaries, neighboring clusters will follow, when nodes and cluster heads for processing information and communication, the cluster head nodes from receiving after the information transmission and fusion information to the adjacent cluster head.

The process can be divided into three stages: the election of cluster head, cluster building and cluster switch and dissolved phase, first for the whole sensor network at time t, not all sensor nodes can detect target, namely not all sensor nodes need to participate in tracking, even participated in the target tracking sensor nodes, each node coordinates to the position of the moving target estimates the contribution of different also. So in order to save energy of the whole sensor network, sensor network to extend the survival time and it is necessary to participate in tracking sensor nodes in the choice of dynamic residual energy as cluster head nodes. Then, from the adjacent nodes of cluster head selection more residual energy of nodes as dynamic temporary cluster of cluster members, and finally in the continuous tracking, select the surplus energy is larger, far away from the original cluster head nodes as the next dynamic temporary cluster of cluster heads, the dissolution of the temporary clusters, become the new cluster head after the selected node, continue to be responsible for tracking moving target, update the confidence value of moving target, choose a new leader nodes, the process is repeated until the moving targets to leave, so can reduce the producing cost of cluster head in tracking, reduce tracking the overall energy consumption, and can avoid due to the large prediction error or target deviation caused by the loss.

All members within the cluster nodes to periodic detected target information, the current time, 
and its node ID to construct a data message is sent to the cluster head nodes, officials after the node to receive data messages, drop it on their own data queue, head node itself as a leader, starts a timer, timer timeout after restart the timer, and processes the data in the queue data message, the message for each of these data, after processing all the data message, target node localization algorithm, the estimation of target location coordinates, and the calculated results and the current time to construct a result message is sent to the management center, handed over to the leader or exit the leader when the leader node, cancel the timer, if received after leader handed over to the other node data messages are forwarded to the new head node.

There are many algorithms of target localization based on sound, by means of sound intensity measurement using weighted centroid localization algorithm for target localization, solved the problem of the simple precision is unstable.

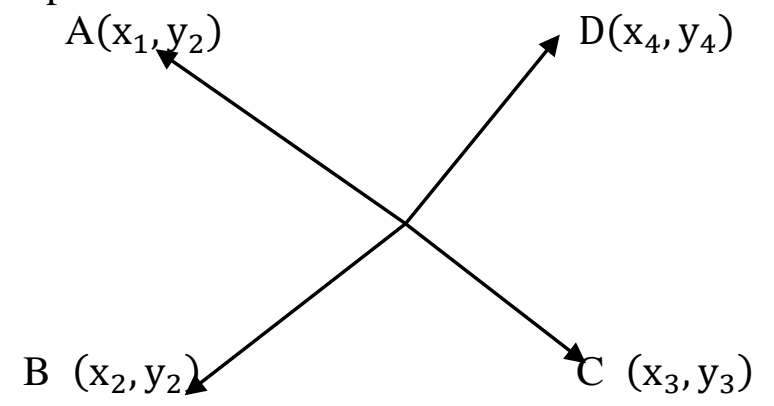

In the diagram, four nodes A, B, C, D coordinates respectively,,,, the distance to the target them respectively,,,, the target coordinates, to beg, in computing, can use the four points of the weighted centroid, namely

$$
\bar{X}=\sum_{i=1}^{n}\left(\omega_{i} \times x_{i}\right) / \sum_{i=1}^{n} \omega_{i} \quad \bar{Y}=\sum_{i=1}^{n}\left(\omega_{i} \times y_{i}\right) / \sum_{i=1}^{n} \omega_{i}
$$

In the formula, that is, $(\bar{X}, \bar{Y})$ o estimate for the weight of the case of a node when calculating the results $\mathrm{I}, \mathrm{n}$ is the number of data messages, said from the intuitive, if the more close to the target, a sensor node is the greater weight should be in the above formula, and should with the inverse of the distance to the target node detected target sound energy and is inversely proportional to the square of the distance of the target, therefore, can use node detect target the square root of sound energy as the weight.

\section{The node hardware design}

The sensor nodes of this system consists of sensor module, processor module, communication module and power supply module, sensor node has three states, active S1: in this state sensor nodes can sense/send/receive information; Monitor state S2: this state node sensing signal environment, not forward data information; Dormant S3: turn off the radio frequency communication, when the state sensing the environment, only minimal energy consumption, considering the reliability of the system, the system selects the JENNIC company fully integrated single chip JN5121 as ZigBee technology solutions.JN5121 the master control chip internal integration with 412 bit ADC, 2 road 11 DAC and the comparator, two application timer and UART, etc., therefore, need to add less peripheral hardware circuit, and can significantly reduce the development effort.

\section{Communication protocol design}

(1) with redundancy check broadcast forwarding mechanism

Communication protocol design of one of the main problem is the broadcast storm problem in wireless communication. When broadcast forwarding redundant copies of the same packet inspection and abandon, can effectively prevent the packets in the network are repeatedly forwarding.

Broadcast forwarding node packets sent by different nodes. If forwarding node again hear the 
same packet sent back from the other nodes, then as a redundant packet discard, record on each node forward recently for a period of time of history, redundant inspection can be realized.This redundancy check mechanism can ensure each node on the same packet forwarding at most once.

(2) to position as the center of the routing protocol

Vehicle tracking system needs to be away from the base station is the feature of the vehicle information through multiple hops forward form sent to a base station, need to design by the agreement to ensure the direction of data transmission.

Based on node positioning itself as a result, the design for the center with location routing protocol, and can realize packets have to spread. Routing policy only forwards more than he can be simply described as node from a base station node packets. As shown in figure 3 using redundancy checking method of centering on the location routing protocol.6 for node distance of the base station distance relationship.2 nodes send packet forwarding path for the node nodes 2-5-1-3 - node 6 , including 3 nodes according to the redundant nodes of packets dropped 4 check mechanism. Routing decisions are easy to implement, as long as the source node is added in the packets from the base station, the forwarding nodes to compare the size of the distance with themselves and the distance between the base station, the routing decisions for the center with location can be realized.

\section{Conclusion}

Vehicle tracking system of wireless sensor network (WSN) this paper puts forward the design scheme of target detection, network communication, based on the system architecture to determine the sensor node design, hardware design, the design of communication protocol, for the establishment of the vehicle tracking system to build solid theoretical basis. Adopting modular design, the use of high performance and high scalability JN5121 chip, the scheme has good versatility and high reliability. In the vehicle positioning monitoring system, has the very strong practicability and convenience, and has certain promotion value.

\section{References}

[1] Wang Shu Yan Yujie, the theory and application of wireless sensor network (WSN), 402-403 (2007).Reference to a book:

[2] Ren Shansi,Li Qun,Wang Haining. Design and Analysis of Sensor Seheduhng Algorithms Under Partial Coverage for Object Detection in Sensor Works[J].IEEE Transactions on Parallel and Distributed Systems,2007,(03):334-350.

[3] kuangxinghong,Location tracking technology in wireless sensor network (WSN) research. Shanghai jiaotong university in 2008 to join collection Get the latest

[4] J.Rayson.Aggregate Towers:Scale Sensitive Visualization and Declutteriong of Geospacial Data .IEEE,1999.

[5] N.Ramanathan,E.Koher,D.Estrin.Towards a debugging system for sensor network.International Journal for Network Managenment ,Jul2005,Volume 15,Issue 4,223-224 